\title{
Damage evolution and fracture events sequence in various composites by acoustic emission technique
}

\author{
A. Bussiba ${ }^{\mathrm{a}, *}$, M. Kupiec ${ }^{\text {a }}, \mathrm{S}$. Ifergane ${ }^{\mathrm{a}}, \mathrm{R}$. Piat $^{\mathrm{b}}$, T. Böhlke $^{\mathrm{b}}$ \\ ${ }^{a}$ Nuclear Research Centre Negev, P.O. Box 9001, Beer-Sheva, Israel \\ ${ }^{\mathrm{b}}$ Institute of Engineering Mechanics, Karlsruhe University (TH), Kaiserstrasse 12, 76128 Karlsruhe, Germany
}

Received 30 May 2007; received in revised form 10 August 2007; accepted 28 August 2007

Available online 18 September 2007

\begin{abstract}
Three composites materials, Glare 2 fiber metal laminates, graphite/epoxy (Gr/Ep) and carbon/carbon (C/C) have been tested mechanically under quasi-static loading in uniaxial and bending modes using uniform and notched specimens. Acoustic emission (AE) technique was utilized in tracking the damage accumulation profile during loading up to fracture in terms of AE counts rate and cumulative. In addition wavelet transforms was used to process AE signals in order to obtain both frequencies and time information on the main failure mechanism and the sequential events during fracture process. This was supported by light and electron microscopies characterizations. The mechanical and acoustical responses were examined with respect to orientation and temperature effects for the Glare 2, exposure temperature effect for the Gr/Ep and porosity degree effect for the C/C. Manifestly, the AE results demonstrate different damage build-up profiles and point to a transition in failure micro-mechanisms with respect to the influence of each parameter (temperature, orientation, and density) on the specific composite tested. For the Gr/Ep, the wavelet transforms indicate the sequence of events in the fracture process, from fiber breaks followed by debonding and ending with matrix cracking. In some cases, it has been observed that the damage accumulation profile in terms of AE resembled the dependency of the crack density versus the strain predicted by a micro-mechanical damage model.
\end{abstract}

(c) 2007 Elsevier Ltd. All rights reserved.

Keywords: A. Layered structures; B. Microstructure; B. Fracture; C. Damage mechanics; D. Acoustic emission

\section{Introduction}

The increasing demand, from the engineering viewpoint, for using advanced composite materials in critical load bearing components in a variety of structures under different loading modes, requires great efforts in various research areas such as development of reliable methodologies for life prediction, characterization of damage evolution and elucidation of fracture micro-mechanisms. These complicated issues are still long term goals, however, in recent years intense activity has been devoted to two different but complementary and linked avenues. One approach is related to

\footnotetext{
${ }^{*}$ Corresponding author. Tel.: +972 86466541 ; fax: +97286567593.

E-mail address: busarie@bezeqint.net (A. Bussiba).
}

the development of damage models in different loading modes such namely quasi-static and cyclic (fatigue), usually based on micro-mechanics [1-3]. On this basis, it predicts stiffness changes in composite materials due to cracking in quasi-static loading and has confirmed the calculated change in the longitudinal Young's modulus due to transverse cracks. Improved models employ mathematical expressions to describe damage evolution, predict changes in the initial material symmetry caused by damage as well as the stiffness changes for all probable crack patterns. In cyclic loading mode, the key feature of the model is that it incorporates delamination associated with transverse cracks in a manner that induces further formation of transverse cracks as delamination grows in fatigue. The model predicts the evolution of transverse crack density and the associated overall elastic moduli changes. The second 
approach is the application of non-destructive testing techniques for the characterization of damage progress and micro-mechanisms of failures in composite materials. Of these methods, AE $[4,5]$ is the most powerful technique due to its link between AE data and fracture mechanics, which makes this relation a very useful tool from the engineering point of view [6]. In this context, $\mathrm{AE}$ can also monitor the damage onset and the damage evolution as well as its profile in real time data, which can assess the structural integrity of the component under loading. Beyond these important engineering considerations, AE can highlight the fundamental damage mechanisms and fracture processes in composites. For the former, events such as fibermatrix interfacial debonding, matrix plastic deformation and cracking, fiber pull-out and fiber-break-up can be characterized by a variety of AE parameters, especially amplitude distribution [7]. For the latter, more refined work utilizes the wavelet transforms to process AE signals in order to obtain both frequencies and time information [8-10]. Fast Fourier Transform (FFT) analysis, points out the dominant frequencies, which are directly related to the main failure mechanism, whereas Short-Time Fast Fourier Transform (ST-FFT) procedure gives the time information and hence the sequential events during fracture process. Briefly, ST-FFT's and Spectrograms allow visualizing and analyzing the data in both a time and frequency domain at the same time. This gives a perspective into how a waveform's frequency content is varying at any particular point or time of the waveform. This simultaneous time and frequency domain can be visualized as a graph with the standard time axis that would be representative of the waveforms length with frequency responses arranged side by side vertically on the time axis. The mathematical form of this function is given in details by [8].

The current study is based on an experimental program, which studied the combined mechanical and acoustical characterizations of three composites materials: fiber metal laminates-Glare 2 [11], Gr/Ep [12] and C/C [13]. The tested composites differ in their design matrixes and fibers, dissimilarities that are reflected in their different mechanical behaviors. The AE data were analyzed in terms of counts rate and cumulative counts while FFT and ST-FFT procedures were also applied for the Gr/Ep composite. In addi- tion, a comparison of the current $\mathrm{AE}$ results with the micro-mechanical model developed for some composites is also suggested and discussed.

\section{Materials and experimental procedures}

\subsection{Materials}

Three composite materials with different matrices were selected, "metallic", polymeric and ceramic. The first-one was Glare 2 (registered trademark of ALCOA) that belongs to the fiber metal laminates family (FMLs) and is used as shielding material against debris and meteors in space stations and as aircraft skins. This material represents a lay-up sequence of three layers (each $0.3 \mathrm{~mm}$ thick) of 2024-T3 Al alloy, and two prepreg (prepreg containing a chemical thickening agent is called a mold-mat) layers of R-glass-fibers (each $0.25 \mathrm{~mm}$ thick) in an epoxy matrix, for a total thickness of $1.4 \mathrm{~mm}$ (Fig. 1a). The material consists of a 0.6 volume fraction of unidirectional fibers in the epoxy resin. At the final stage of processing, it was poststretched by $0.4 \%$ in order to achieve residual compressive stress in the metal and residual tensile stress in the fiberresin layer. The second-one was $\mathrm{Gr} / \mathrm{Ep}, 1.5 \mathrm{~mm}$ in thickness, primarily oriented to aircraft and space structures, torsion applications and as a reflector support truss. This polymeric reinforced composite with a lay-up orientation of $90^{\circ} / 80^{\circ}$ was processed from magnamite prepreg tape T1000/556/917/070 (Fig. 1b). The continuous fibers were high strength filaments, surface treated, designated T1000 and the resin was amine-cured epoxy, designated 556/ $917 / 070$. Generally, the surface treatment is intended to improve the composite shear and transverse tensile strength. The third-one was $\mathrm{C} / \mathrm{C}$ also recognized as carbon fiber reinforced ceramic matrix of carbon (CFRC), used for aeronautics and space technology due to its high strength at temperatures up to $1800^{\circ} \mathrm{C}$. This composite was produced by isothermal, isobaric chemical vapor infiltration (CVI). This process for fabrication of CFRC materials consists of the synthesis of carbon particles from hydrocarbon gas and their deposition on carbon fiber felts (CCKF 1001, Sintec, Germany) made of randomly oriented fibers (where the fiber volume content is about $12 \%$ (of the total volume)

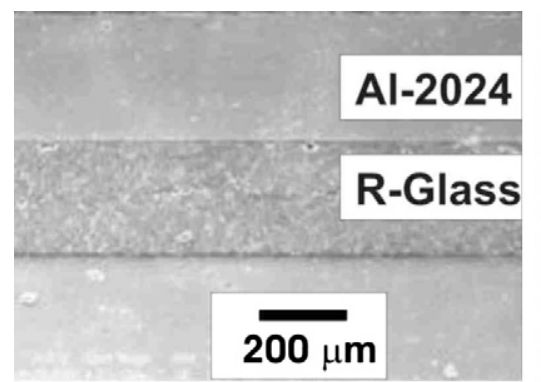

a

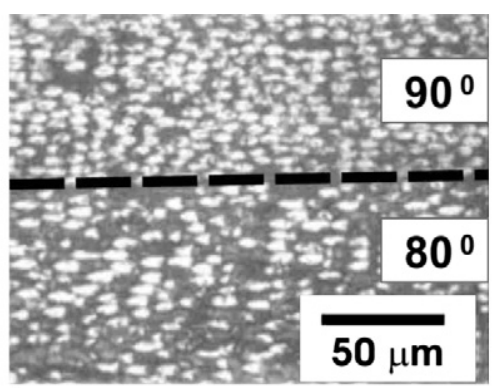

b

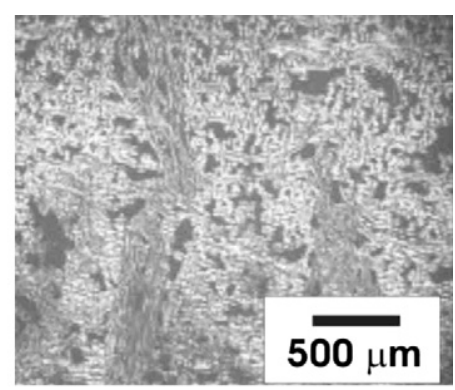

C

Fig. 1. Microstructures of the tested composites: (a) glare 2, (b) Gr/Ep, and (c) C/C. 
and the diameter of the fibers is of the order of $10 \mu \mathrm{m}$ ) (Fig. 1c). The felts were infiltrated under different conditions (time of infiltration) to obtain matrices with high or low porosity. Infiltration times for the composites were $120 \mathrm{~h}$ for low porosity (LP) (obtained to be 15\%) and $45 \mathrm{~h}$ for high porosity (HP) (obtained to be $30 \%$ ). The process was performed at a temperature of $1095^{\circ} \mathrm{C}$ and total pressure of $30 \mathrm{kPa}$. In this process, the carbon particles deposit in the form of a pyrolytic carbon matrix around the fibers. This matrix has a layered structure and consists of pyrolytic carbon with different textures $[14,15]$.

\subsection{Experimental procedures}

For Glare 2, effects of orientation and temperature were characterized by means of mechanical and acoustic response. For this, uniaxial properties were determined by tensile tests using a uniform flat specimen in longitudinal $(L)$ and transverse $(T)$ with respect to fiber orientation. Anisotropic fracture resistance was assessed by means of fracture toughness testing. Here, fatigue pre-cracked compact tension specimens (CTS) were tested longitudinally (LO) and transversely (TO). More experimental details are reported elsewhere [16].

For the Gr/Ep with lay-up of $90^{\circ} / 80^{\circ}$, the effect of exposure temperature was examined by both mechanical and acoustical testing. Here, flat specimens, with orientations parallel to the fibers, were tested in uniaxial tensile tests. The exposure temperature and time were selected to be $396 \mathrm{~K}$ and 14 days, respectively. In order to follow the sequence of the main micro-fracture events during fracture of this composite, three additional designed tensile specimens were prepared in order to characterize the acoustic profile of fiber breaks, matrix cracking and debonding. For the latter, the specimen was cut such that the fibers were oriented at $10^{\circ}$ to the loading direction. This loading stimulates shear stress which controls the debonding process.

For the $\mathrm{C} / \mathrm{C}$, the effect of porosity degree was explored by flexural tests of uniform and notched bars while monitoring AE activity. Full details of both mechanical testing and more on the AE set-up are reported elsewhere [17]. Table 1 summarizes the test conditions for the three composites including the number of tested specimens.

All the mechanical testing was conducted on a computerized servo-hydraulic machine and the $\mathrm{AE}$ was simultaneously tracked with load, strain and crack opening displacement (COD) signals. The AE tests were performed at Gabi Shoef LTD company using computerized AE system MISTRAS 2001 manufactured by PAC. Miniature resonant sensor model $9223 \mathrm{M} 3$ with dimension of $9 \mathrm{~mm} \times 2 \mathrm{~mm}$ (diameter $X$-height) was used with typical frequency range of $50-600 \mathrm{kHz}$. The sensor was fixed to the specimens using a mechanical device and using silicone grease coupling. Pre-amplification was $40 \mathrm{~dB}$ and ambient noise was filtered using a threshold of $30 \mathrm{~dB}$ and a 100 $1200 \mathrm{kHz}$ bandwidth plug-in filter. In addition, preliminary study was done in order to minimize the AE signals generated from friction and local damage from stress concentrations at the loading points.

A calibration procedure was performed in order to select the appropriate parameter (gain threshold) while eliminating the undesired $\mathrm{AE}$ signals. The $\mathrm{AE}$ system allows the processing of a variety of parameters such as counts, energy, rise time, amplitude, average frequency and so on from the AE waveforms. Preliminary measurements determined the acquisition parameters for the current composite materials (PDT $=50 \mu \mathrm{s}$; HDT $=1000 \mu \mathrm{s}$; $\mathrm{HLT}=300 \mu \mathrm{s}$ ), at a sampling rate of $10 \mathrm{MHz}$. In addition, stress wave emissions were monitored continuously for further analysis using FFT procedures. Here, the characteristic frequency $\left(f_{c}\right)$ was determined for a better under-

Table 1

Test conditions for the tested composites (the dimensions are in $\mathrm{mm}$ )

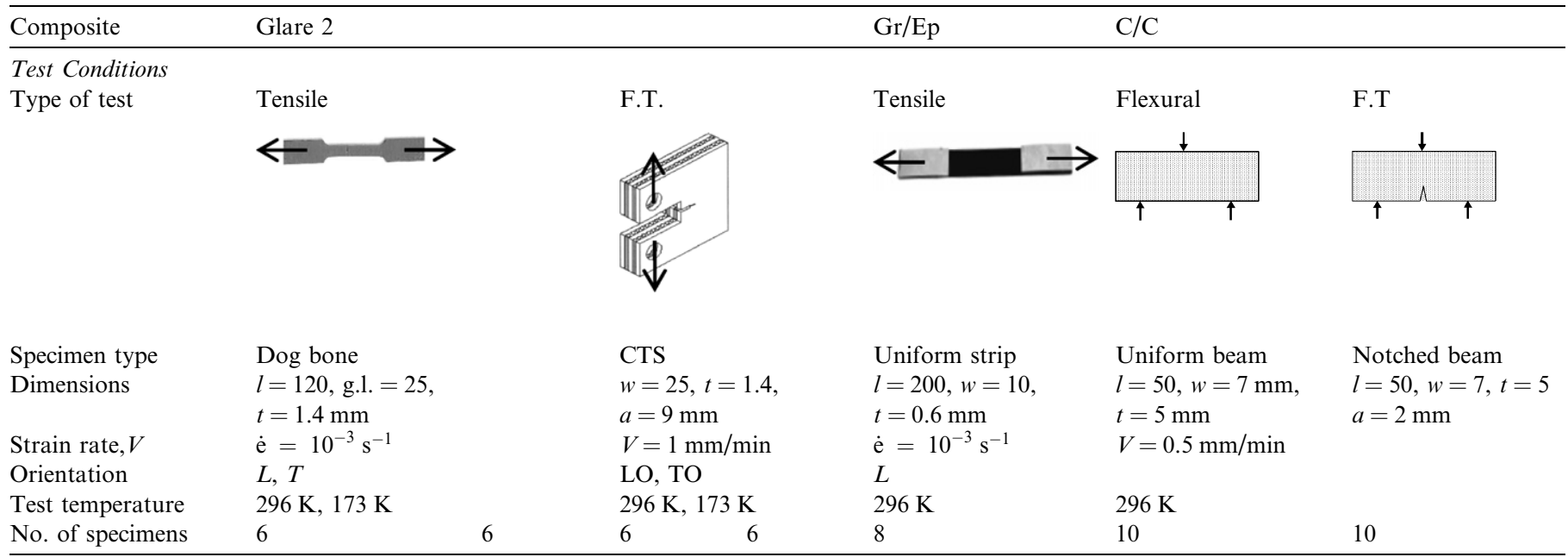

$l$, length; g.l., gauge length; $t$, thickness; $a$, crack length; $w$, width; F.T., fracture toughness; $V$, velocity. 
standing of the damage micro-mechanisms controlling the fracture. Following this, ST-FTT was also performed to obtain both frequencies and time information. These procedures can highlight the damage evolution sequence during loading up to fracture. Finally, light and scanning electron microscopies were used while paying special attention to the important features of fracture of the specific composite. For example, for Glare 2, fiber-break density with stress, fracture modes transition of the epoxy resin with temperature and adhesion of the interface metal/epoxy resin. For Gr/Ep, the focus was on the effect of exposure temperature on the interface fiber/resin and the change in the energy dissipation during fracture of the resin. For $\mathrm{C} / \mathrm{C}$ more has been done in order to track the crack initiation sites, crack profiles and damage features accompanying the main crack, with regard to the porosity. In addition, the main failure micromechanisms were classified including micro cracking in the multilayers.

\section{Experimental results}

\subsection{Glare 2}

\subsubsection{Effect of temperature on the uniaxial mechanical properties}

Fig. 2 depicts the influence of test temperature on tensile stress-strain curves for $L$ orientation tracked by AE. As shown, the AE profile changes as the temperature drops.
At $296 \mathrm{~K}$ (Fig. 2a), the AE counts emerge immediately after the initiation of plastic deformation and increase steeply up to fracture. In terms of cumulative counts, an exponential type profile is mostly observed (Fig. 2b). Completely different AE behavior was noticed at $173 \mathrm{~K}$ (Fig. 2c), where AE counts were first detected at the early stage of loading with alternating appearance up to fracture. This change in AE counts rate is reflected also in terms of cumulative counts (Fig. 2d) where three stages of damage have been detected: stage I with step type profile, stage II that follows exponential behavior of damage accumulation and stage III which is characterized by a linear tendency with a moderate slope. This variation in the AE response is emphasized by the macroscopic mode fracture where at $173 \mathrm{~K}$ the metal layers split away from the resin/fiber layers and the shear-type fracture at $296 \mathrm{~K}$ changes to normal fracture at $173 \mathrm{~K}$. More on the correlation between the failure micro-mechanisms with respect to the temperature and the damage build-up in terms of AE will be discussed later.

\subsubsection{Effect of temperature and orientation on the fracture toughness}

Fig. 3 illustrates the effect of test temperature on the load and AE activity versus COD curves for the sharp cracked specimens in the LO. As shown, the damage evolution at the crack tip accumulates differently at $296 \mathrm{~K}$ as compared to $173 \mathrm{~K}$, reflected by dissimilar AE responses in terms of counts rate (Fig. 3a and c) and cumulative
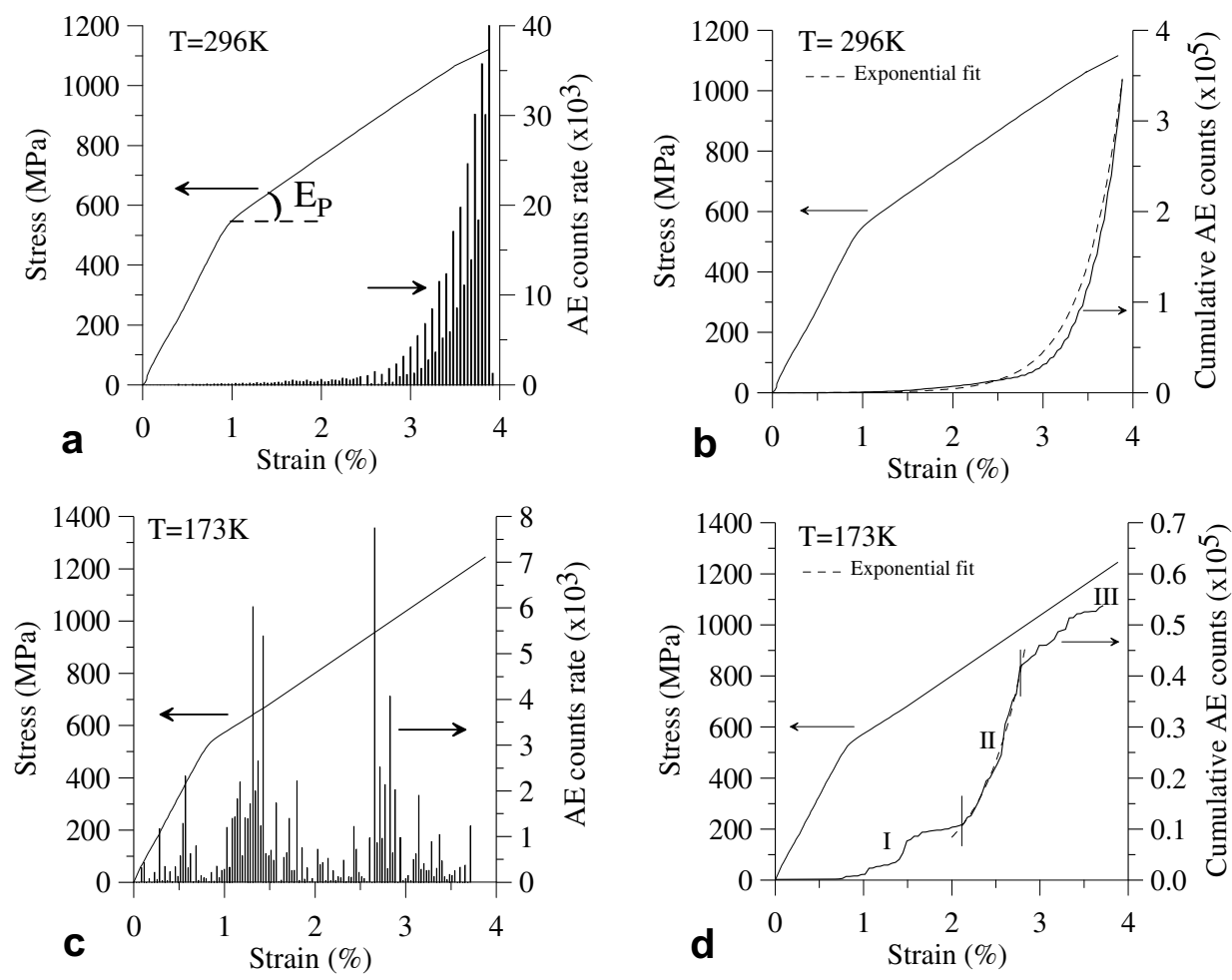

Fig. 2. Effect of temperature on the mechanical and acoustical response for the Glare 2 in $L$ orientation in terms of counts rate and cumulative counts (a and b) $296 \mathrm{~K}$ (c and d) $173 \mathrm{~K}$. 

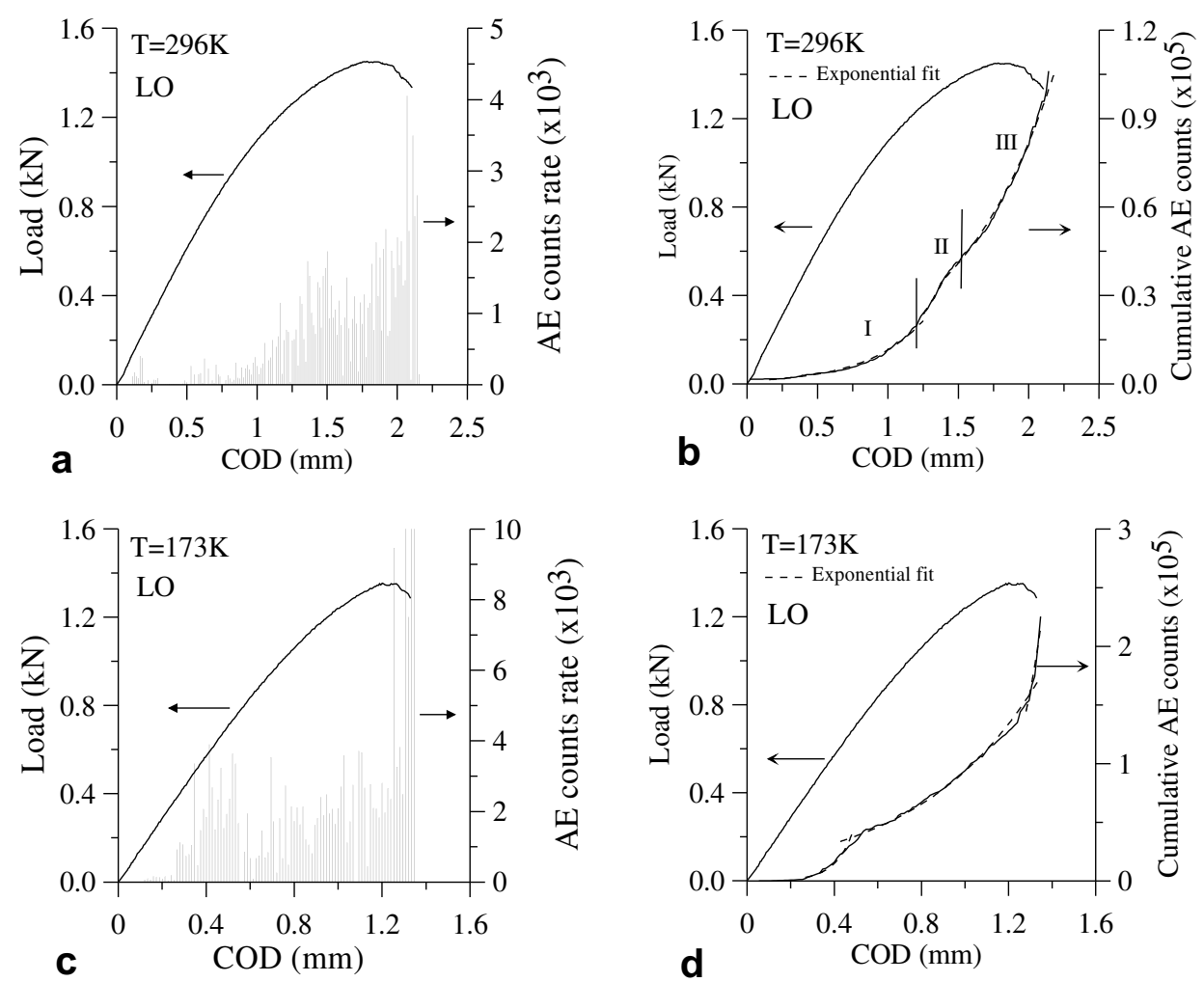

Fig. 3. Effect of temperature on the mechanical and acoustical response for the notched Glare 2 in LO in terms of counts rate and cumulative counts (a and b) $296 \mathrm{~K}$, (c and d) $173 \mathrm{~K}$.
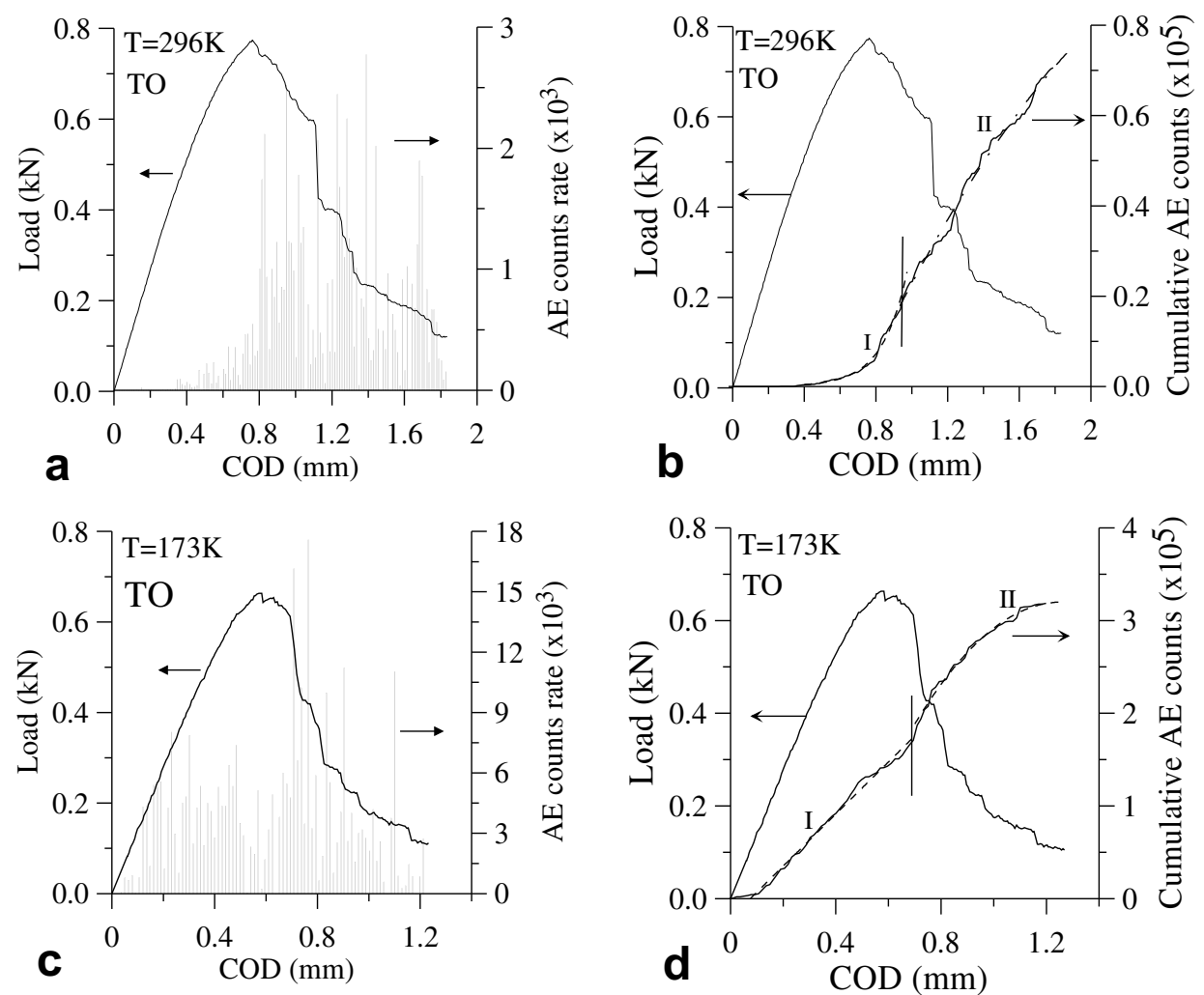

Fig. 4. Effect of temperature on the mechanical and acoustical response for the notched Glare 2 in the TO in terms of counts rate and cumulative counts (a and b) $296 \mathrm{~K}$, (c and d) $173 \mathrm{~K}$. 
counts (Fig. 3b and d). At 296 K (Fig. 3a), AE was noticed at the early stage of loading which increases with the initiation of plastic deformation and intensified later on during the slow crack growth process up to final fracture. At $173 \mathrm{~K}$ (Fig. 3c), the AE counts rate were characterized by nearly the same level from low load up to nearly fracture point with an alternate type profile. The latter AE pattern is related to alternating delamination growth and arrest processes up to final failure. In cumulative display, three stages were observed with an exponential type fit for each stage. As shown, stage II at $173 \mathrm{~K}$ is the dominant one which was characterized by delamination crack growth and crack arrest processes alternately which are being suppressed at $296 \mathrm{~K}$. The occurrence of such extrinsic phenomena is reflected also by the higher toughness at $173 \mathrm{~K}$ $\left(45 \mathrm{MPa} \mathrm{m}^{1 / 2}\right)$ as compared to low one at $296 \mathrm{~K}$ $\left(37 \mathrm{MPa} \mathrm{m}^{1 / 2}\right.$ ). Regardless of test temperature, no crack growth occurred; instead, cracking developed at the $\mathrm{Al}$ alloy layer and propagated towards the load axis. Namely, the metal failed in shear mode, while the fibers remained nearly intact.

Fig. 4 illustrates typical load and AE versus COD curves for the notched TO specimen. As shown, the crack tip damage accumulates differently at $296 \mathrm{~K}$ and $173 \mathrm{~K}$, as reflected by the different AE responses and intensity. At $296 \mathrm{~K}$ (Fig. 4a), AE activity begins at initiation of plastic deformation and intensifies later on during the slow crack growth process, which is accompanied by multiple pop-in events. At $173 \mathrm{~K}$ (Fig. 4c), the AE shape differs substantially when compared to that at $296 \mathrm{~K}$ and is characterized by nearly constant counts rate from the early stage of loading up to the point of deviation from linearity. In cumulative display, transition from exponential to parabolic occurs at the initiation of crack growth for $296 \mathrm{~K}$ while for $173 \mathrm{~K}$, evolution from linear to parabolic behavior is observed. The change in the AE pattern due to the temperature effect is emphasized by different near crack tip damage evolutions up to crack initiation, as shown in Fig. 5. At low temperature (Fig. 5a), cracks due to delamination process are initiated and arrested at the interface (see arrows); these delamination cracks act as a shielding source and delay the formation of macro-cracks. At $296 \mathrm{~K}$ (Fig. 5b), almost no delamination cracking occurs and the damage is accumulated as the load increases.

\subsection{Graphitelepoxy}

Fig. 6 illustrates the effect of exposure temperature on the mechanical and acoustical response of Gr/Ep. As shown (Fig. 6a and b), the unexposed (UE) composite behaves in a linear manner, however in terms of AE; there is a transition in the AE activity at a stress of about $2500 \mathrm{MPa}$. This change is seen in terms of AE counts rate (Fig. 6a) and emphasized further in the cumulative counts presentation (Fig. 6b) where the evolution of the damage is changed from a linear to an exponential type profile. In contrast to a smooth curve of the UE composite, in the exposed $(E)$ one several localized stress relaxations occur (Fig. 6c). This phenomenon initiated at a stress of about $1500 \mathrm{MPa}$ (although minor ones appeared at lower stresses as manifested by abrupt increases in AE counts rate) and its density occurrence increases as it approaches the fracture stress. The linear part of the cumulative curve (Fig. 6d) was suppressed due to the localized stress relaxation followed by an increase in the AE counts, before exponential type accumulation of damage became dominant. The change in the mechanical behavior as well as in the $\mathrm{AE}$ activity due to the temperature effect is well reflected on the fracture surface (Fig. 7). It can be seen that the adhesion of epoxy resin to the fiber becomes poor (Fig. 7b) as compared to the UE (Fig. 7a), which affects the debonding stress. This change in the interface fiber/ resin properties is more accentuated in the fibers oriented at $10^{\circ}$ to loading direction (Fig. $7 \mathrm{~d}$ ) where more interface cracking is developed as compared to adherent interface for the UE (Fig. 7c). Moreover, for specimens where the fibers were perpendicular to the loading orientation (not shown here), the interface fracture is completely different for the $\mathrm{E}$ one as compare to the UE. The brittle cracking with tongue-like appearance for the $\mathrm{E}$ is emphasized as compared to a smooth one for the UE.

\subsection{Carbon/carbon composite}

\subsubsection{Flexural mechanical properties}

Fig. 8 depicts a comparison of mechanical and acoustical responses of the $\mathrm{C} / \mathrm{C}$ composite with different porosities, in terms of AE counts rate (Fig. 8a) and cumulative counts (Fig. 8b) in stress and strain displays. As shown,

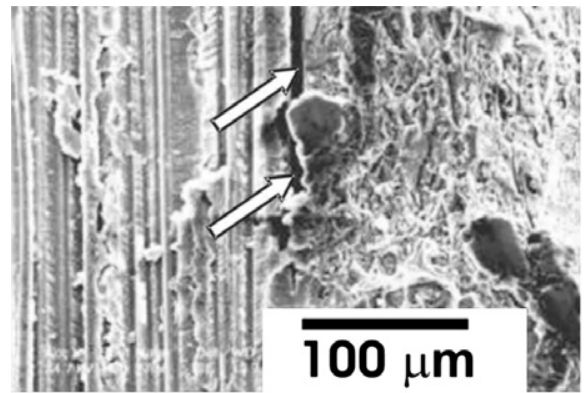

a

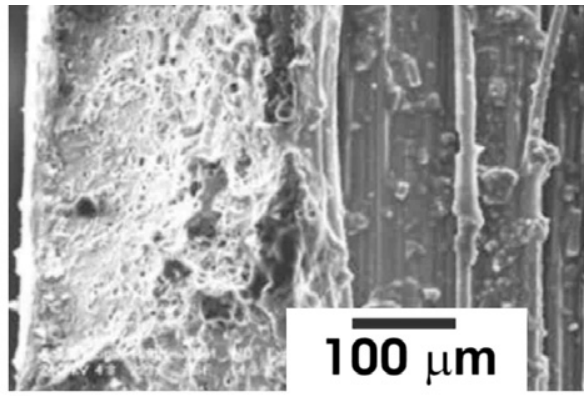

b

Fig. 5. Microscopic cracking features in the notched TO Glare 2 specimens: (a) $173 \mathrm{~K}$; (b) $296 \mathrm{~K}$. 

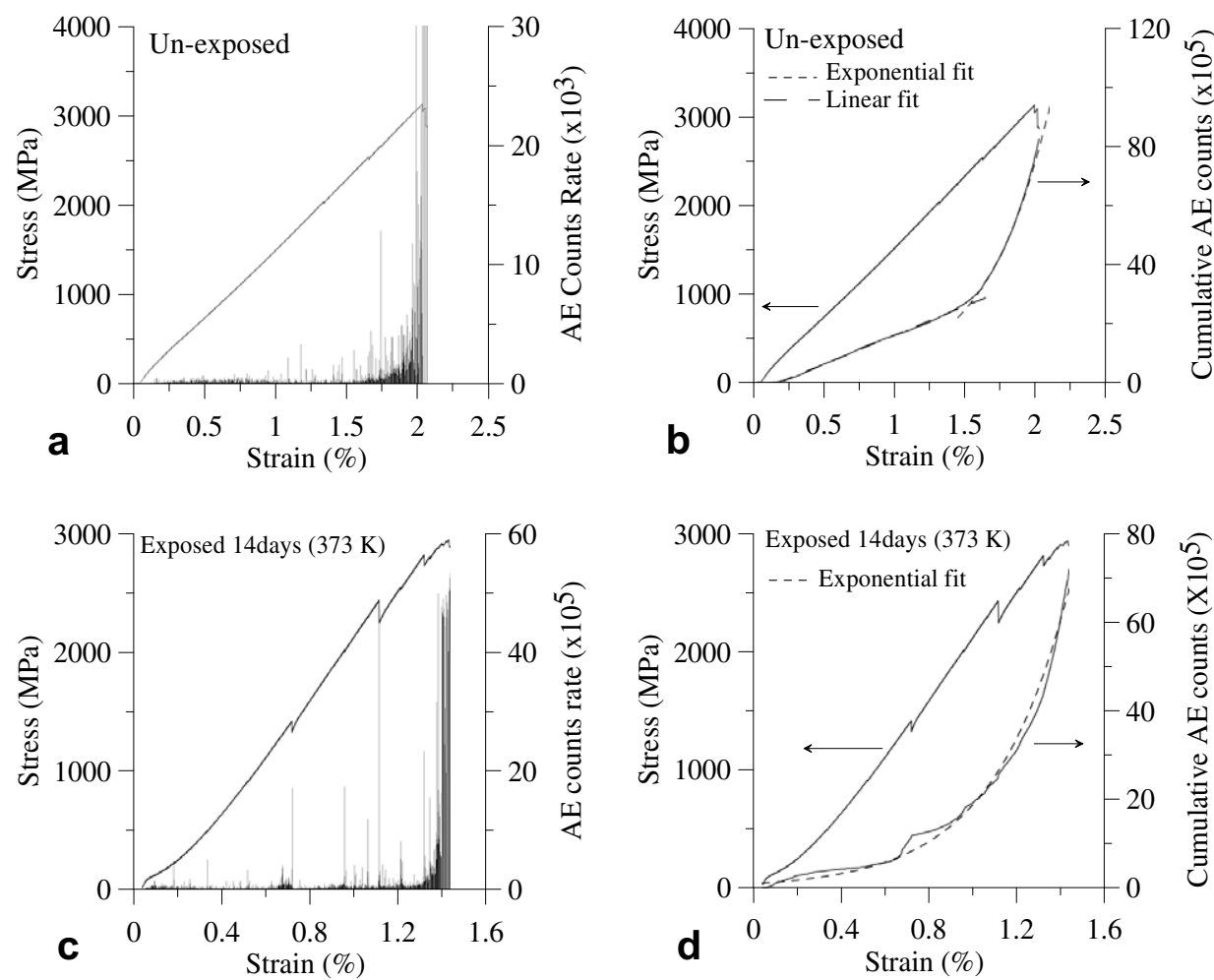

Fig. 6. Stress and AE vs. strain for the Gr/Ep UE composite (a and b), E composite (c and d).
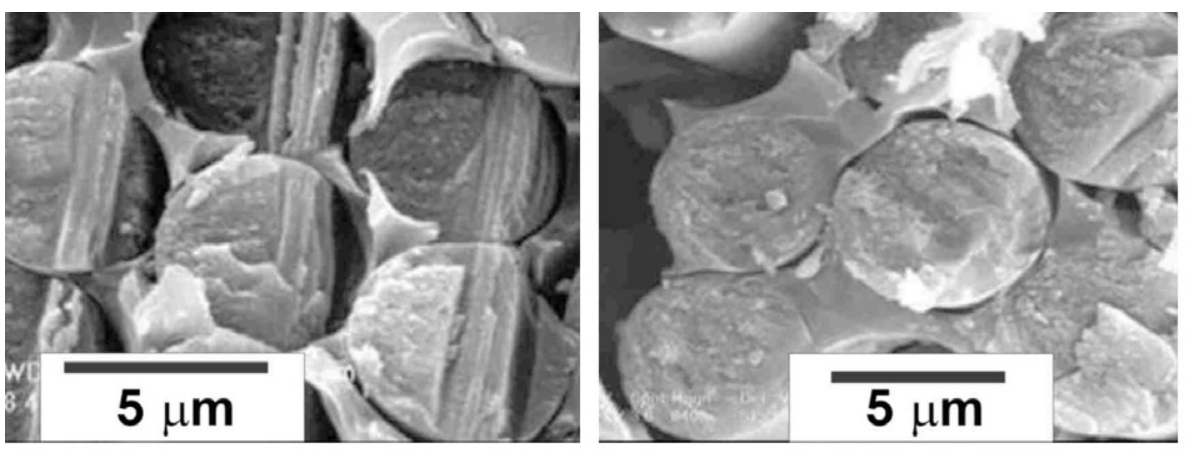

a

b
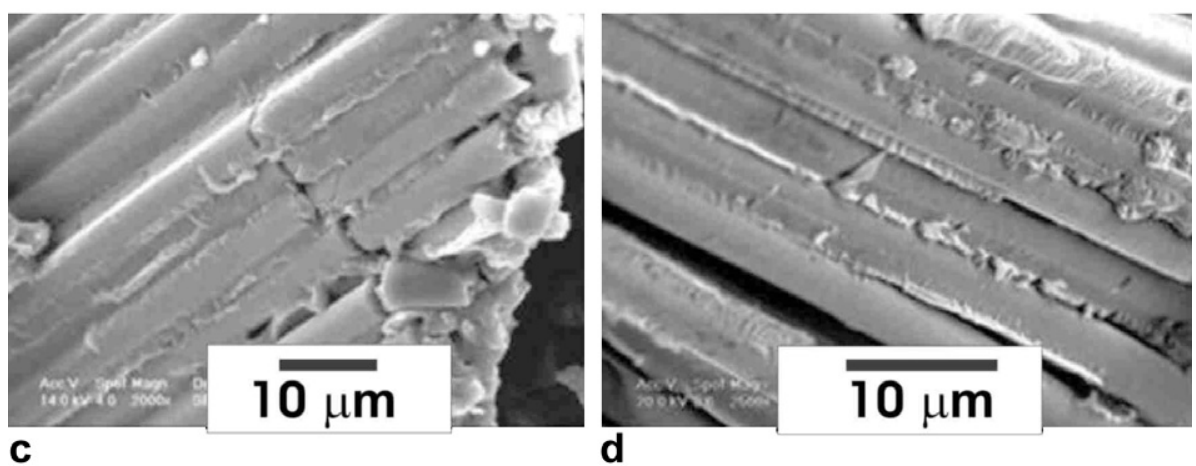

Fig. 7. The change in fracture features due to the temperature for the Gr/Ep: (a) UE, (b) $E$, (c) $80^{\circ} \mathrm{UE}$, (d) $80^{\circ} E$.

in both composites, there exists a threshold strain $\left(e_{\text {th }}\right)$ and corresponding threshold stress $\left(\sigma_{\mathrm{th}}\right)$ for the onset of $\mathrm{AE}$ activity (Fig. 8b). This finding was also detected by English [5] for PVC/glass-fiber and by Siron et al. [18] for filler - added coal-tar pitch-based $\mathrm{C} / \mathrm{C}$ composites. It can be seen that $e_{\mathrm{th}}$ for the onset of $\mathrm{AE}$ activity is higher (almost twice) for the LP as compared to that for the HP. This AE finding indicates that no damage is initiated below this threshold 

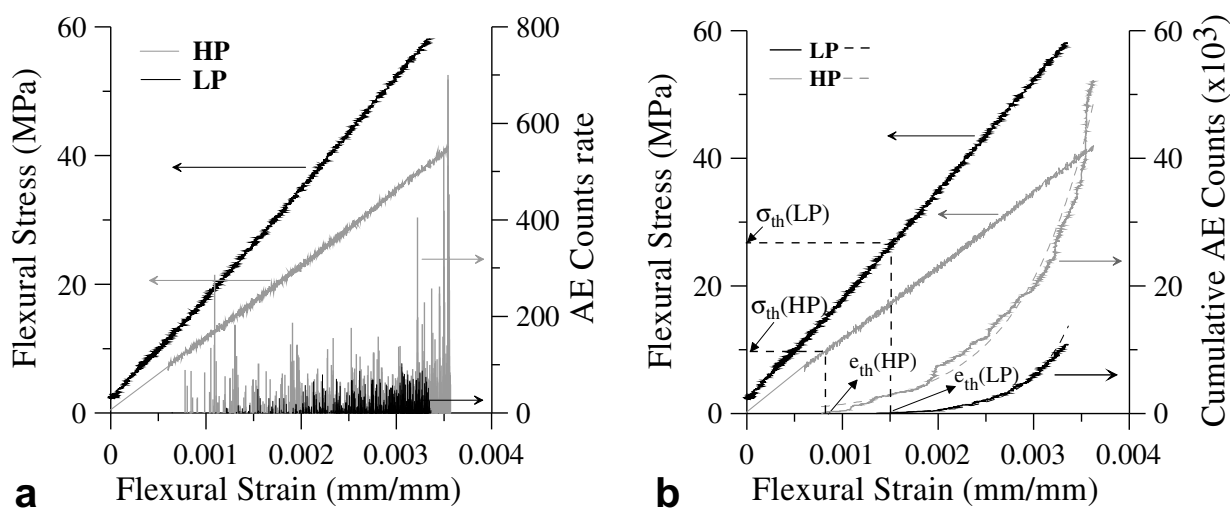

Fig. 8. Comparison of mechanical and acoustical responses for both $\mathrm{C} / \mathrm{C}$ composites in terms of (a) AE counts rate (b) cumulative counts.

strain in this specific composite. This parameter can be an important one in many applications from modifications in manufacturing processes to damage degree evaluation due to service. The damage accumulation profile in terms of $\mathrm{AE}$ is demonstrated in Fig. $8 \mathrm{~b}$ by the presentation of cumulative counts. While linear mechanical response is well depicted, an exponential type of contour is obtained for the AE response. So, the damage evolution by AE data in such composites follows the exponential function of the strain.

Fig. 9 shows typical failure micro-mechanisms observed near the outer surface of the fractured beam in both composites. In general, for both composites, the main micro-mechanisms can be pointed out: (1) fracture of fibers surrounded by carbon multilayers that are parallel or nearly parallel to the loading direction. For the HP (Fig. 9a), the result of the incomplete process is reflected in the peeling of the layers due to weak interfaces. This can be compared to more complete layers with strong interfaces as seen for the LP (Fig. 9b). (2) Fracture of the interface resulting from the intersection of two multilayer growths when the fibers (or the felt) are perpendicular to the loading direction (Fig. 9c). (3) Fracture of the carbon matrix that exhibits low energy fracture surfaces. (Fig. 9d, right part of the photo). The characteristic multilayer fracture and the fibers of the LP are also depicted in Fig. 9d.

\subsubsection{Fracture resistance properties}

Fig. 10 describes the typical mutual responses of two notched composites, in terms of AE counts rate (Fig. 10a
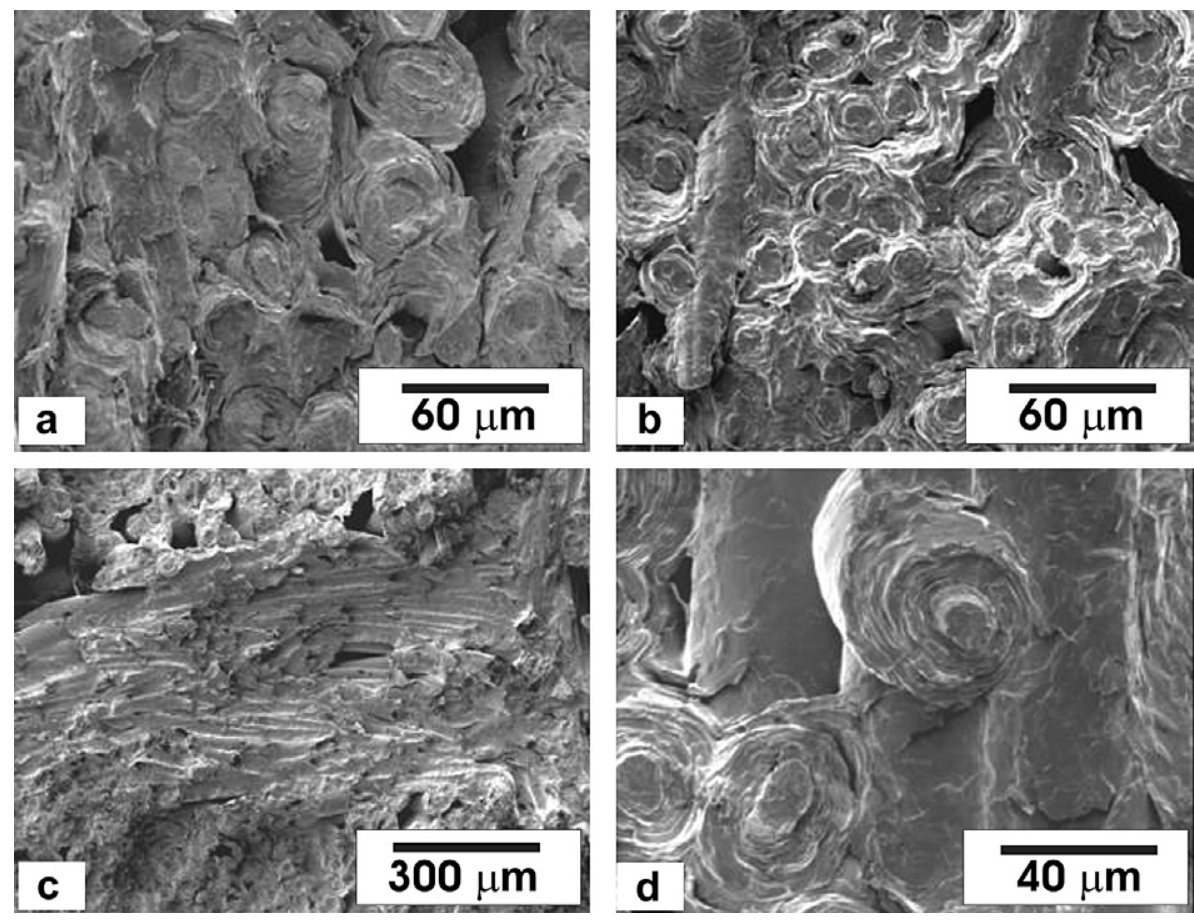

Fig. 9. SEM micrographs of failure micro-mechanisms observed in flexural test of C/C composite: (a) fibers and the multilayered in HP, (b) fibers and the multilayered in LP, (c) fracture of matrix/fibers interface, and (d) fracture of the matrix (matrix = multilayered). 

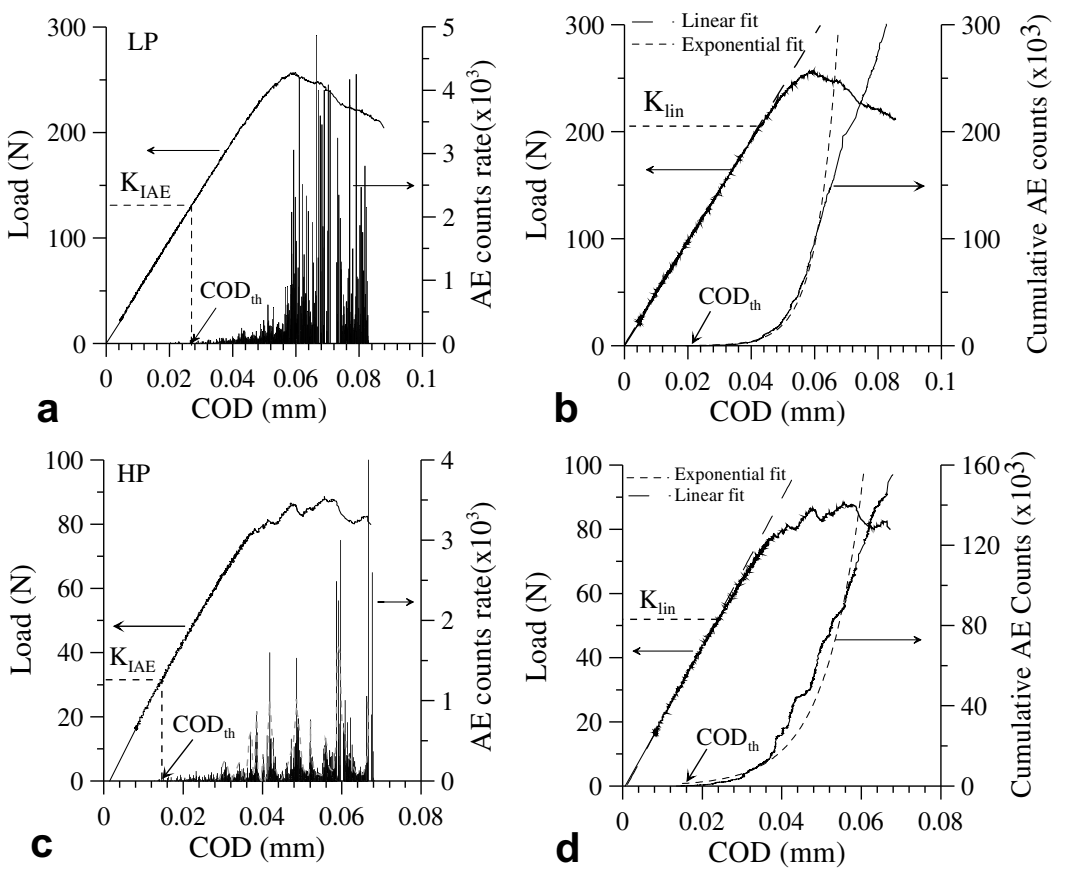

Fig. 10. Load, AE counts rate (a and c) and cumulative (b and d) vs. COD for C/C composite in LP and HP.

and c) and AE cumulative counts (Fig. 10b and d). Again, there exists a threshold $\mathrm{COD}_{\text {th }}$ (alike to threshold strain) for the onset of AE activity in both composites. The existing of such $\mathrm{COD}_{\text {th }}$ which indicates that any damage not yet occur in the specimen is also reported by Woo and Choi [19] for unidirectional glass-fiber/epoxy resin prepregs. The corresponding $K$, designated by $K_{\mathrm{IAE}}$ is almost half of the LP $K_{\mathrm{IC}}$ whereas for the HP it is only a third of the $K_{\text {IC }}$. The macroscopic deviation from linearity in the load-COD curve is depicted in Fig. 10b and c designated by $K_{\text {Ilin. }}$ which indicates an intensification of the AE count rates resulting from the transition in damage accumulation. This change is accentuated in the HP composite (Fig. 10c) where cycle type profile is observed up to the fracture with an increasing amplitude of alike sinusoidal wave. Moreover, discontinuities in the load-COD curve are noted with the progress of the fracture process. This type of curve is very similar to those of quasi-brittle metallic materials that exhibit the pop-in phenomena (abrupt rapid crack growth followed by localized crack arrest). Here, the origin of this mechanism, namely alternating crack growths and arrest events up to fracture, is the presence of pores that act as barriers for the advancing crack. The secondary mechanism is related to the crack branching phenomena (see later on), which affects the nominal remote stress intensity factor. These two mechanisms are well suppressed in the LP composite and manifested in nearly smooth curves with no indication of macroscopically discontinuous crack growth (Fig. 10a). The AE findings support the mechanical behavior, while the AE counts rate increases steeply with the load.

Finally, Fig. 11 illustrates characteristic fracture modes for both composites. Fig. 11a and b emphasize the fracture of the fibers near the notch tip in HP with an enhancement of multilayered microcracking (Fig. 11b). The fracture of the fibers and the matrix cracking with low energy fracture surface for the LP are shown in Fig. 11c and d, respectively.

\section{Discussion}

The current study highlights the great potential of the $\mathrm{AE}$ technique in fundamental and engineering aspects with some linking. For the former, monitoring of damage evolution with relation to the failure micro-mechanisms and might point out the fracture sequences. For the second, it may indicate the damage degree and reveal some critical or threshold mechanical parameters for undamaged condition during service. As such, the AE results illuminate different damage build-up profiles and point to a transition in failure micro-mechanisms during loading with respect to the influence of each parameter (temperature, orientation, and density) on the specific composite tested.

For the Glare-2, the main failure micro mechanism in tensile mode of $L$ orientation at $296 \mathrm{~K}$ is associated with fiber-break-up, which becomes dominant as the remote load increases, resulting in a second low modulus, $E_{\mathrm{p}}$ (see Fig. 1a). This claim is based upon a metallographic thickness cross-section taken from a specimen loaded to 0.75 $0.85 \sigma_{\text {UTS }}$, which shows a high density of broken fibers (Fig. 12a). This sequence of events is reflected by an exponential type of continuous damage accumulation. At low temperatures, distinct damage progression and fracture mechanisms have been observed. The controlled fracture mechanism evolved with the debonding process at the metal/resin-fiber interface, a zone that is weak due to a 

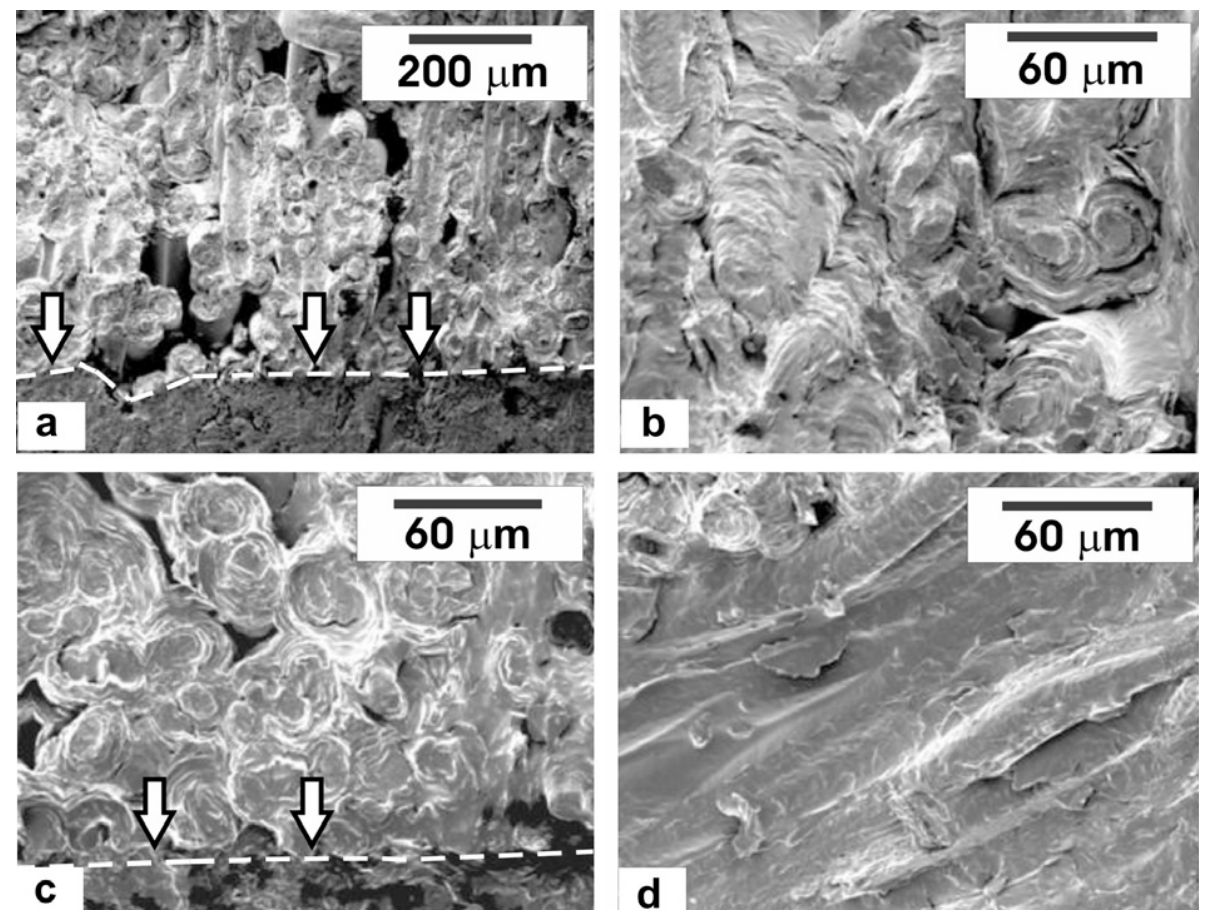

Fig. 11. SEM images of the main fracture modes developed during crack growth in $\mathrm{C} / \mathrm{C}$ composite: (a) fibers break near notch tip in HP, (b) microcracking at the multilayers, (c) fibers break near notch tip in LP, and (d) matrix cracking.

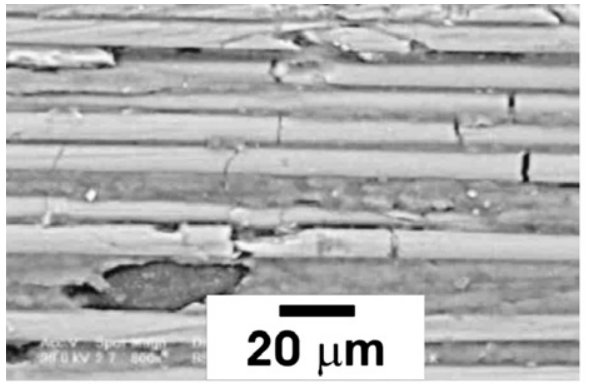

a

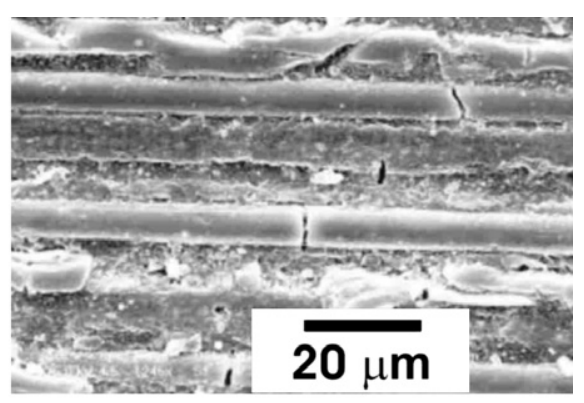

b

Fig. 12. Fibers break-up with different densities developed in Glare 2 composite during uniaxial tensile test up to 0.75 of the fracture stress at (a) $296 \mathrm{~K}$ and (b) $173 \mathrm{~K}$.

mismatch in some physical properties. This type of damage occurs before sufficient local stress reaches a level for fiberbreak-up. Thus, the fracture starts at some discrete zones along the interface which coalesce near the maximum stress with a low density of fiber breaks (Fig. 12b). At the point of fracture, the remaining intact areas failed and the metal layers separated from the fiber/resin layers. This sequence of fracture events is characterized by none continuous damage accumulation. In cumulative display three stages were distinguished, an indication of transition in failure mechanisms where at stage II is characterized by delamination cracking (due to debonding) and arrest, followed by fiber breaks alternately up to near fracture.

The interface metal/resin-fiber in Glare 2 plays an important role in case of crack growth resistance. Thus, temperature effect on the strength of this interface is easily seen by the different $\mathrm{AE}$ responses and hence different type of damage evolution. At $296 \mathrm{~K}$ in LO, the major damage at the crack tip process zone is developed at the metal layers accompanied by some damage at the interface and the fiber/resin layers. This order of the fracture sequence is displayed by monotonical increases in AE counts rate as the crack deviates towards the loading direction (no crack growth occurs normal to the loading direction). At $173 \mathrm{~K}$, short-transverse delamination (perpendicular to the crack plane) takes place due to mismatch in thermal expansion between the metal and the resin. Consequently, the crack tip stress intensity is reduced and further loading is needed to continue the fracture process. The great propensity towards delamination cracking with some damage at the metal layers is responsible for none continuous growth (alternately) in the AE counts rate.

The brittleness behavior of the interface fiber/resin of the $\mathrm{TO}$ at low temperature exposure in addition to the 
short-transverse delamination cracking, contribute to the almost linear profile of cumulative $\mathrm{AE}$ counts from the early stage of loading. This behavior is in contrast to the gradual mode of damage for the entire in stage I at $296 \mathrm{~K}$.

Similar to the Glare 2, the interface fiber/resin of the $\mathrm{Gr} /$ Ep is responsible for the acceleration in damage development and loss of the integrity in this composite under sustained load. The long exposure to temperature alone causes degradation in the efficiency in the strength bond between the fiber and the resin in addition to some changes in the structure of the resin which makes it more brittle. These two effects influence its mechanical response under monotonic loading when events of localized load relaxation occur at the early stage of loading as compared to smooth curves for the unexposed composite. As complementary, the $\mathrm{AE}$ findings show a transition in damage accumulation from linear/exponential curve for the unexposed composite to the almost exponential one which is an indication of enhanced damage growth.

Clearly, the influences on the controlled micro-mechanisms on fracture processes are being reflected on different types of damage accumulation in terms of AE results. This finding becomes more significant with the information on the sequence fracture events as will be discussed briefly for the Gr/Ep. As mentioned, ST-FFT analysis decomposes any waveform into frequency bands and local time. The following example emphasizes this in Gr/Ep where the sequential events during the fracture process are explored with this procedure. Fig. 13a-c show the characteristic frequencies $\left(f_{\mathrm{c}}\right)$ acquired by FFT for matrix cracking, debonding and fiber-break events, respectively which have been obtained by testing the individual constituents. Fig. 13d illustrates the $f_{\mathrm{c}}$ pattern obtained from using FFT analysis on a typical $\mathrm{AE}$ waveform collected during fracture of the Gr/Ep. As shown, three $f_{\mathrm{c}}$ are displayed where the lowest represents the matrix cracking, the highest is the fiber-break and the middle one represents the debonding process. Fig. 13e shows the integrated ST-FFT analysis of the AE waveform with the time information extraction for the $f_{\mathrm{c}}$ shown in Fig. 13a-c. Consequently, the sequence of events in the fracture process of the $\mathrm{Gr} / \mathrm{Ep}$ is the following: fiber breaks followed by debonding and ending with matrix cracking. This order is very reasonable since the failure strain of the carbon fibers is less than the matrix. Once the fibers fail, the normal stress applied to the fibers is released causing debonding failure followed by matrix cracking. The statement on the dominant frequencies and hence the fracture sequences is still limited and must not be over interpreted due to the number of influences on the $f_{\mathrm{c}}$, such as the size and shape of the specimen (resonant frequencies!) and by the characteristics of the sensors.

From engineering sense, the damage degree during service can be extracted from the damage evolution profile obtained during loading in terms of cumulative AE counts. This statement is being supported by suggested micromechanical models which have been developed to estimate the damage degree in composites. A non-linear damage behavior was presented by Wang [20] using a mechanical damage model and by Talreja [1], who predicted that crack density is an exponential function of the strain for bilinear
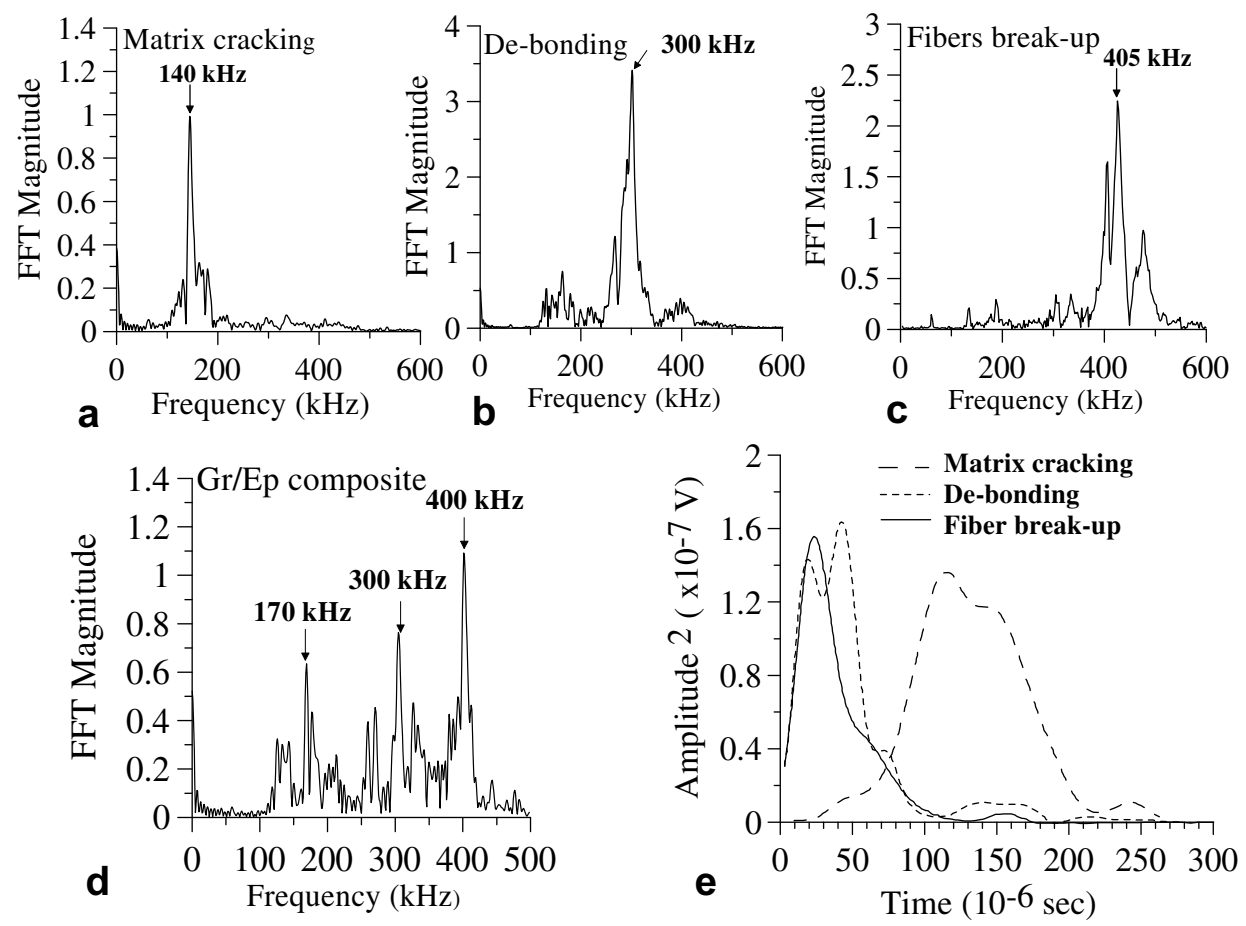

Fig. 13. FFT and ST-FFT of the constituents and whole Gr/Ep: (a) $f_{\mathrm{c}}$ of the matrix cracking, (b) $f_{\mathrm{c}}$ of the debonding process, (c) $f_{\mathrm{c}}$ of the fibers break-up, (d) $f_{\mathrm{c}}$ of the whole Gr/Ep composite, and (e) ST-FFT with time frequency and time information. 
stress-strain behavior in composites laminates. This exponential dependency was obtained in terms of cumulative $\mathrm{AE}$ counts in tensile of Glare- 2 at $\mathrm{L}$ orientation, from the nearly elastic/plastic region up to fracture. Tensile of unexposed $\mathrm{Gr} / \mathrm{Ep}$, revealed exponential form at the later stage of loading, while for the exposed specimen, this type of behavior was obtained almost from the initial loading. For the $\mathrm{C} / \mathrm{C}$ under bending mode, exponential growth of damage progress is developed from a threshold strain no matter of the density level. In addition, in case of loading of notched bar, the exponential profile is still preserved up to the initiation of macroscopic crack growth. So we can conclude that such profile of damage is controlled for some composites in specific orientation and loading modes. Thus, this similarity between the results predicted by micro-mechanical models and the experimental AE data suggests a supplementary and important approach in quantifying the damage degree in composites material. Finally, the existing of mechanical thresholds, whether strain or $\mathrm{COD}$, indicates that no damage occurs, a proof which is very important for structures where no damage is allowed during service.

\section{Acknowledgements}

R. Piat gratefully acknowledges the financial support received by the German Research Foundation (DFG, Center of Excellence 551 in Research on "Carbon from the gas phase: elementary reactions, structures and materials").

\section{References}

[1] Talreja R. Continuum modeling of the development of interlaminar cracking in composite laminates. In: Salama K, Ravi-Chandar K, Taplin DMR, Rama Rao P, editors. Advances in fracture research: conference Proceedings of the seventh International Conference on Fracture. Houston, Texas; March 20-24, 1989. p. 2191-99.

[2] Nagendra VA, Talreja R. A micromechanics based model for predicting fatigue life of composites laminates. Mat Sci Eng 2000;A285:303-13.

[3] Nagendra VA, Talreja R. A mechanistic model for fatigue damage evolution in composite laminates. Mech Mat 1998;29(2):123-40.

[4] Pollock AA. Acoustic emission inspection. Metals handbook, 9th ed., vol. 17. ASM International; 1989.
[5] English LK. Listen and learn: AE testing of composites. Mater Eng May 1987; TR103-75-6/87.

[6] Huang M, Jiang L, Liaw PK, Brooks CR, Seeley R, Klarstrom DL. Using acoustic emission in fatigue and fracture materials research. $\mathbf{J}$ Miner Metals Mater Soc 1998;50(11): <www.tms.org/pubs/journals/ $\mathrm{JOM} / \mathrm{jom} \cdot \mathrm{html} />$.

[7] Xingmin Z, Xiong Y. Investigation of damage mechanisms in selfreinforced polyethylene composites by acoustic emission. Comp Sci Tech 2006;66(3-4):444-9.

[8] Ni QQ, Kurashiki K, Iwamoto M, AE technique for identification of micro failure Modes in CFRP composites. Mater Sci Res Int 2001;7(1):67-71.

[9] Groot PJ, Wijnen PA, Jansen BF. Real time frequency determination of acoustic emission for different fracture mechanisms in carbon/ epoxy composites. Comp Sci Tech 1995;55(4):405-12.

[10] Woo SC, Choi NS. Analysis of dominant frequencies of glass-fiber/ aluminum laminates during acoustic emission measurement. Key Eng Mat 2006;321-323:901-6.

[11] Lambert M, Schneider E. Shielding against space debris. A comparison between different shields: the effect of materials on their performances. Int J Impact Engng 1995;17:477-85.

[12] Shin ES. Effects of hydrostatic pressure on the torsional shear behavior of Graphite/ Epoxy composites. J Compos Mater 1992;26(4):462-85.

[13] Manocha LM. High performance carbon-carbon composites. Sādhanā 2003;28(1-2):349-58.

[14] Reznik B, Hüttinger KJ. On the terminology for pyrolytic carbon. Carbon 2002;40(4):621-4.

[15] Reznik B, Gerthsen D, Hüttinger KJ. Macro- and nanostructure of the carbon matrix of infiltrated carbon fiber felts. Carbon 2001;39(2):215-29.

[16] Bussiba A, Kupiec M, Ifergan S. Fracture of fiber metal laminate Glare 2. Advances in cryogenic engineering (materials), vol. 46. 2000; p. $159-66$.

[17] Bussiba A, Kupiec M, Piat R, Böhlke T. Fracture characterization of $\mathrm{C} / \mathrm{C}$ composites under various stress modes by monitoring both mechanical and acoustical responses, Carbon, accepted for publication.

[18] Siron O, Chollon G, Tsuda H, Yamauchi H, Maeda H, Kosaka K. Microstructural and mechanical properties of filler-added coal-tar pitch-based $\mathrm{C} / \mathrm{C}$ composites: the damage and fracture process in correlation with AE waveform parameters. Carbon 2000;38(9): 1369-89.

[19] Woo SC, Choi NS. Analysis of fracture process in single-edgenotched laminated composites based on high amplitude acoustic emission. Compos Sci Technol 2007;67(8-9):1451-8.

[20] Wang TJ. In: Teoh SH, Lee KH, editors. Proceedings of the joint FEFG/ICF international conference on fracture of engineering materials \& structures. Singapore; August 6-8, 1991. p. 251-56. 